\title{
Performance Study of IEEE 802.15.4 under OPNET Modeler for Wireless Sensor Networks
}

\author{
Rania Khadim ${ }^{\star 1}$, Mohammed Erritali ${ }^{2}$, Abdelhakim Maaden ${ }^{3}$ \\ ${ }^{1}$ Laboratory of Mathematics and Applications, Faculty of Sciences and Techniques, \\ Sultan MoulaySlimane University \\ ${ }^{2}$ TIAD laboratory, Departement of Computer Sciences, Faculty of Sciences and Techniques, \\ Sultan Moulay Slimane University \\ *Corresponding author, e-mail: khadimrania@gmail.com ${ }^{1}$, m.erritali@usms.ma
}

\begin{abstract}
The IEEE 802.15.4 protocol has recently been adopted as a communication standard for low-rate wireless personal area networks (LR-WPANs) due to its low data rate, low power consumption and low cost of Wireless Personal Area Networks. This protocol is quite flexible for a wide range of applications if appropriate tuning of its parameters is carried out. Importantly, the protocol also provides real-time guarantees by using the Guaranteed Time Slot (GTS) mechanism. Indeed, the GTS mechanism is quite attractive for Wireless Sensor Network (WSN) applications. The main objective of this paper is to investigate the performance of BPSK (Binary phase shift keying) and QPSK (Quadrature phase shift keying) modulation techniques. Investigations have been reported to compare the performance of the two modulation schemes. Here modulation schemes have been identified which gives significant performance improvement over the other based on network output load, energy consumption and power reception at the WPAN devices. The results have been presented Pan Coordinator, GTS and Non GTS End Device (CAP device) of Wireless Sensor Network (WSN).
\end{abstract}

Keywords: IEEE 802.15.4, LR-WPAN, WSN, BPSK, QPSK

Copyright $\odot 2015$ Institute of Advanced Engineering and Science. All rights reserved.

\section{Introduction}

In the recent years, wireless sensor communication sector has experienced a conspicuous rapid growth. Several short range wireless technologies have been developed as an answer to the increasing demand for portable and flexible connectivity.

Wireless sensor network kind of technologies have followed suit owing to the adoption of services where data is shared and exchanged with requirements for such technologies driven by the need for larger data throughput, lower Bit Error Rate (BER), higher Signal to Noise Ratio (SNR), minimum end to end delay etc. In addition to the upsurge in the deployment of IEEE 802.11 based wireless local area networks (WLANs), few complementary low-power and low cost technologies among which IEEE 802.15.4 are establishing their place on the market as enablers of the emerging wireless sensor networks (WSNs).

The IEEE 802.15.4 standard was specifically developed to address a demand for low power, low bit rate connectivity towards small and embedded devices. Furthermore the standard is trying to solve some problems that were inadequately taken into account by Bluetooth technology.Despite the intense research and standardization tasks performed over the last years on maximizing the network output load and minimizing the energy consumption, still many open issues are to be dealt with before wireless sensor networks can be utilized on large scale.

Researchers have carried out the research in various areas and a lot of work on 802.15.4 has been reported by the various researchers [1-5]. Some have investigated various performance issues like: Delay; Throughput evaluation of GTS mechanism [1]. While some have investigated distributed active control technique for IEEE 802.15.4 wireless sensor network [3]. Few others have worked on power efficient radio configuration infixed broadband wireless networks [4]. Researchers have also studied adaptive algorithm for mapping channel quality information to modulation and coding schemes [5].

The IEEE 802.15.4 standard, which is specifically designed for low data rate, low power consumption, and low complexity applications, is well suitable for the requirements of wireless 
sensor networks (WSNs). It is characterized by a maximum range of a few hundred meters and a low flow [6].

The Multiple kinds of topologies are supported by IEEE 802.15.4 such as: star, peer-topeer and cluster-tree. The IEEE 802.15.4 standard specifies the physical layer and the MAC layer, it operate either in a non-beacon-enabled or beacon-enabled WSNs mode. The operation mode is determined by a single central controller termed the PAN coordinator. Coordinator can bind its channel time using super-frames. A super-frame, which is bounded by two successive beacons, can have an active portion and an optional inactive portion. The active portion can be composed of a Contention Access Period (CAP) and an optional Contention-Free Period (CFP). To communicate during the CAPs, any node should compete with other nodes using a slotted carrier sense multiple access/ collision avoidance (CSMA/CA) [7].

In this paper we propose the comparison of two different modulation schemes BPSK(Binary phase shift keying) and QPSK(Quadrature phase shift keying) with the use of Micaz [8] as a battery model to determine the suitability of the scheme according to the type of the device in the network. Here the performance metrics like: network output load, energy consumption and power reception at the radio receiver have been considered for their comparative study.

\section{The Super-frame Structure}

A super-frame is composed of an active portion where nodes can communicate with each other and optionally followed by another inactive portion where nodes are in sleep mode. The Beacon Interval (BI) is the time duration between two successive beacons. The super-frame Duration (SD) is the total time duration of theCAP, CFP (GTS) and a Beacon. The Super-frame duration doesn't include the inactive period.

The Beacon Interval $(\mathrm{BI})$ and the Super-frame Duration (SD) are determined by two parameters, the Beacon Order (BO) (Equation 1) and the Super-frame Order (SO) (Equation (2)), respectively $[9,10]$.

$\frac{B I=\text { aBaseSuperFrameDuration } * 2^{B O}[\text { symboles }]}{0 \leq B O \leq 14}$

$\underline{S D=\text { aBaseSuperFrameDuration } * 2^{S O}[\text { symboles] }}$ $0 \leq S O \leq B O \leq 14$

As shown in the Figure 1, the active portion of the super-frame is divided into 16 equally-sized time slots where the first time slot is occupied by the beacon frame used for network synchronization. The active portion consists of three parts: the beacon, Contention Access Period (CAP) and Contention Free Period (CFP).

Communication between devices during the CAP is a kind of competition using slotted CSMA/CA mechanisms in order to access the medium.

Where the super-frame includes a CFP period, the coordinator ensures access to the medium for some nodes. For this, the CFP period is divided into different parts composed of Guaranteed Time Slots (GTSs).

Note that the Network association requests and GTS request are used just in the CAP period. Each GTS consists of an integer multiple of time slots CFP and there may be up to 7 GTS in a CFP [11, 12].

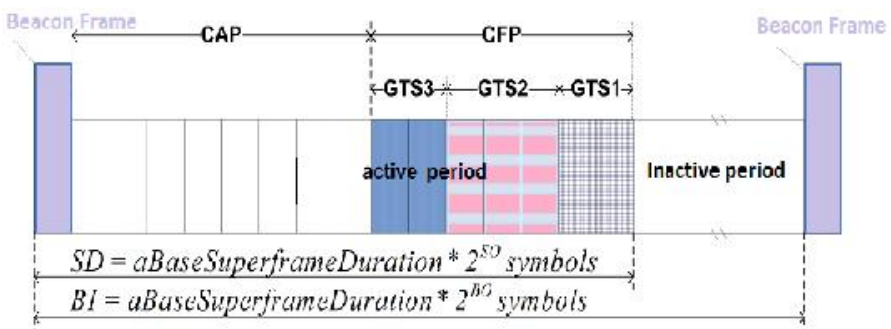

Figure 1. The Super-frame Structure 


\section{Senarios Description}

The simulation model implements physical and medium access layers defined in IEEE 802.15.4 standard. The OPNETModeler 14.5 is used for developing 802.15.4 wireless sensor network.

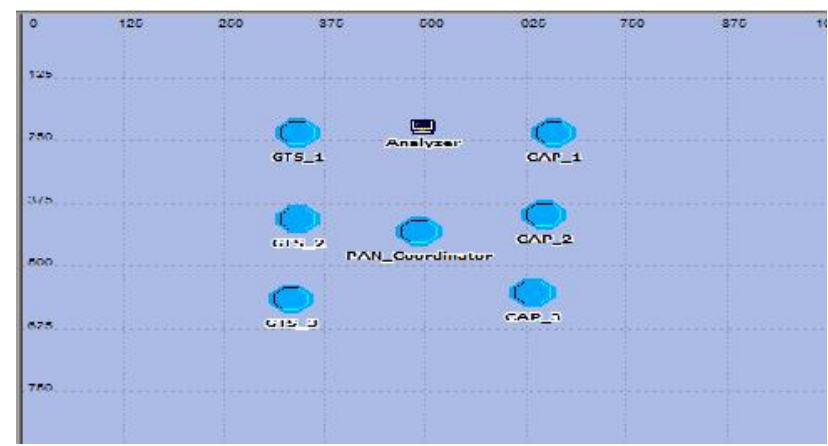

Figure 2. Network Scenario for BPSK and QPSK

Figure 2 shows the Scenario of QPSK(Quadrature phase shift keying) and BPSK (Binary phase shift keying) that contains one PAN Coordinator, one analyzer and six"end devices" out of which tree are Guaranteed Time Slots (GTS) enabled and rest are non GTS devices.

The two different Scenarios have been created with two different modulation formats like: BPSK and QPSK. Following parameters as shown in the Table 1 have been set for the said scenarios:

Table 1. Parametric values for PAN Coordinator, GTS and Non GTS End Device in BPSK and

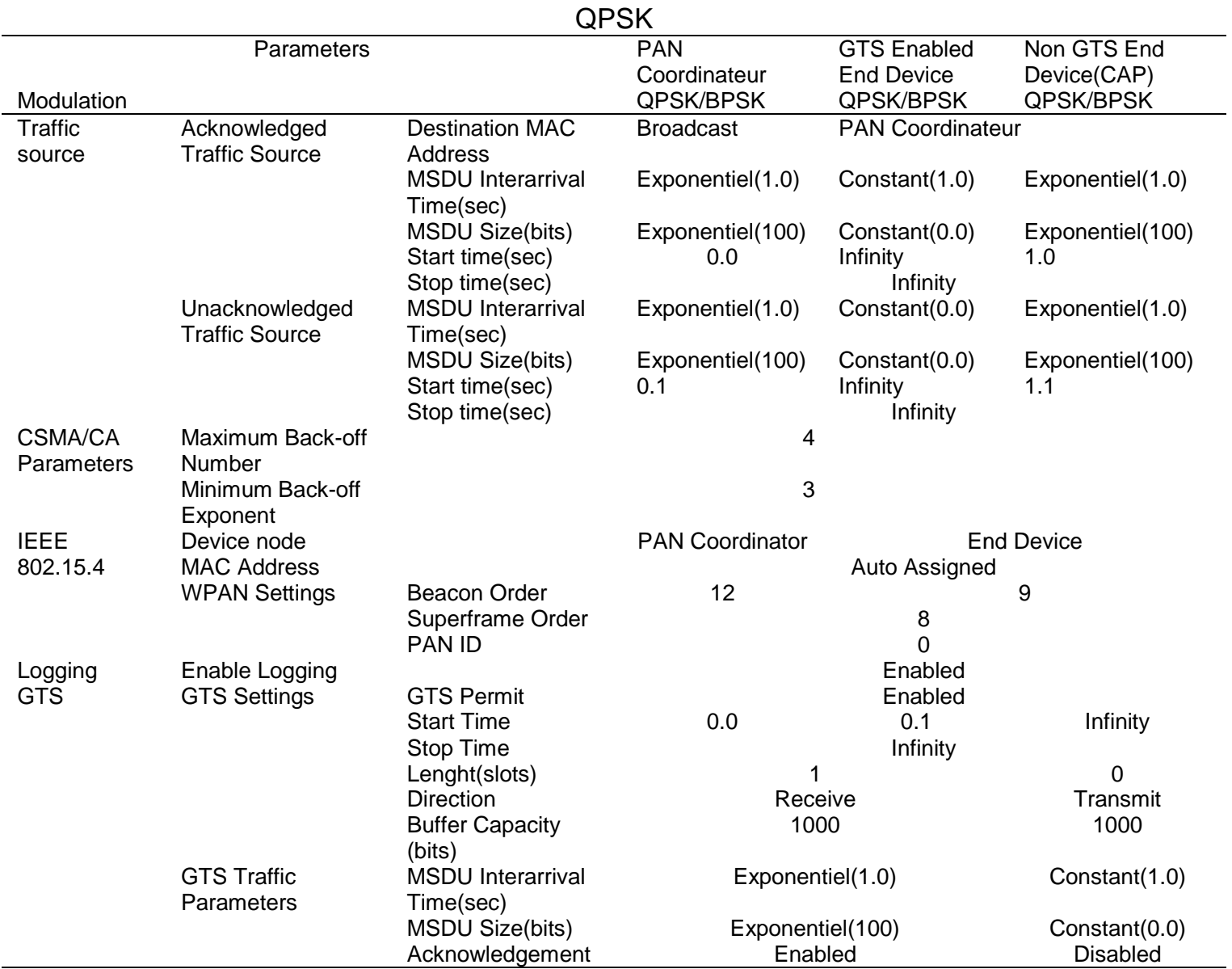

TELKOMNIKA Vol. 16, No. 1, October 2015: $98-105$ 


\section{Results \& Analysis}

The simulation has been caried this section out for the two different scenarios of WPAN: Quadrature phase-shift keying QPSK and Binary phase-shift keying BPSK. In this section results for energy consumption by the battery and power reception of the radio receiver have been presented and discussed for different types of devices in wireless sensor networks.

\subsection{Network Output Load}

\subsubsection{PAN Coordinator}

Figure 3 indicates that the peak value of network output load at the PAN_Coordinatoris 456, $19863 \mathrm{bits} / \mathrm{sec}$ and 451,559322 bits/sec for QPSK and BPSK respectively, and after 19, 8 seconds the value decreases to 51, 25 bits/sec for QPSK and 38, 05 bits/sec for BPSK.

It is observed that the network output load is higher in case of QPSK because by using this modulation we can achieve high SNR (Signal to Noise Ratio) thus we can be sure of less noise in the channel during transmission.

Also it is observed that load is minimum in case of BPSK due to its very low SNR that it provides high immunity to noise as compare to the signal floating through the medium.

Also BPSK and QPSK have similar bit error rate (BER) but the BPSK is able to modulate only $1 \mathrm{bit} / \mathrm{sec}$ but by using QPSK, we can transmit twice amount of data on the same channel with the same bandwidth.

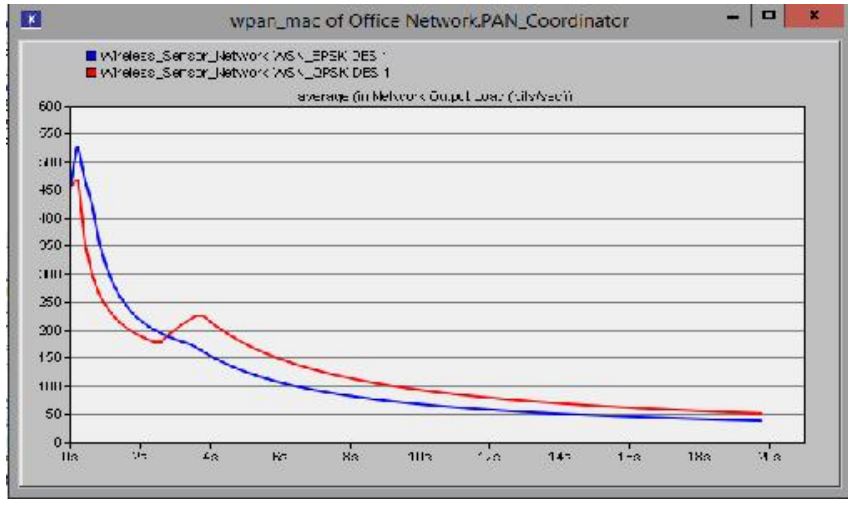

Figure 3. Netwok Output Load at the PAN_Coordinator

\subsubsection{GTS End Device}

Figure 4 depicts that the network output load at the GTS End Device is: $602,789396 \mathrm{bits} / \mathrm{sec}$ and $602,654867 \mathrm{bits} / \mathrm{sec}$ for QPSK and BPSK respectively in the beginning of the simulation.And after for QPSK and BPSK the value decreases to $12 \mathrm{bits} / \mathrm{sec}$.

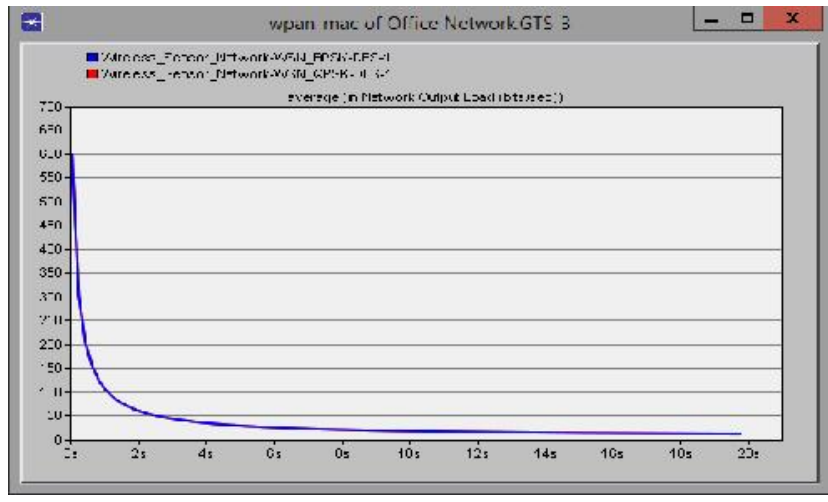

Figure 4. Netwok Output Load at the GTS End Device 


\subsubsection{CAP End Device (Non GTS End Device)}

Figure 5 shows that the network output load at the Non GTS End Device is: 328,634921 bits/sec and 234,698158 bits/sec for QPSK and BPSK respectively in the beginning of the simulation and after 19, 8 seconds the value decreases to 124,45 bits/sec for QPSK and $62,65 \mathrm{bits} / \mathrm{sec}$ for BPSK.

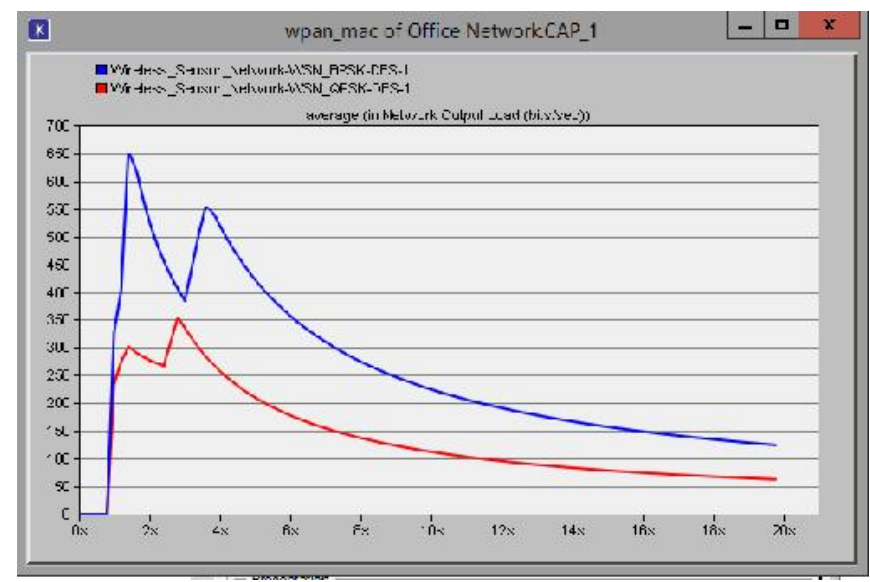

Figure 5. Netwok Output Load at the Non GTS End Device

\subsection{Energy Consumed}

Figure 5 shows that the network output load at the Non GTS End Device is: 328,634921 bits/sec and 234,698158 bits/sec for QPSK and BPSK respectively in the beginning of the simulation and after 19,8 seconds the value decreases to $124,45 \mathrm{bits} / \mathrm{sec}$ for QPSK and $62,65 \mathrm{bits} / \mathrm{sec}$ for BPSK.

\subsubsection{PAN_Coordinator}

Figure 6 indicates that the peak value of the energy consumed by the PAN Coordinator is 0.00147992 and 0.00166499 joules for the QPSK and BPSK respectively.

It is observed that battery energy consumption is minimum in case of QPSK due to its constant envelope that allows to use amplifiers in their best performance area that often corresponds to a non-linear operation, also QPSK uses four points on the constellation diagram equispaced around a circle with four phases that's why the energy spreads on a bounded interval of four states (phases).

For BPSK modulation energy consumption depends on the constellation point's position.

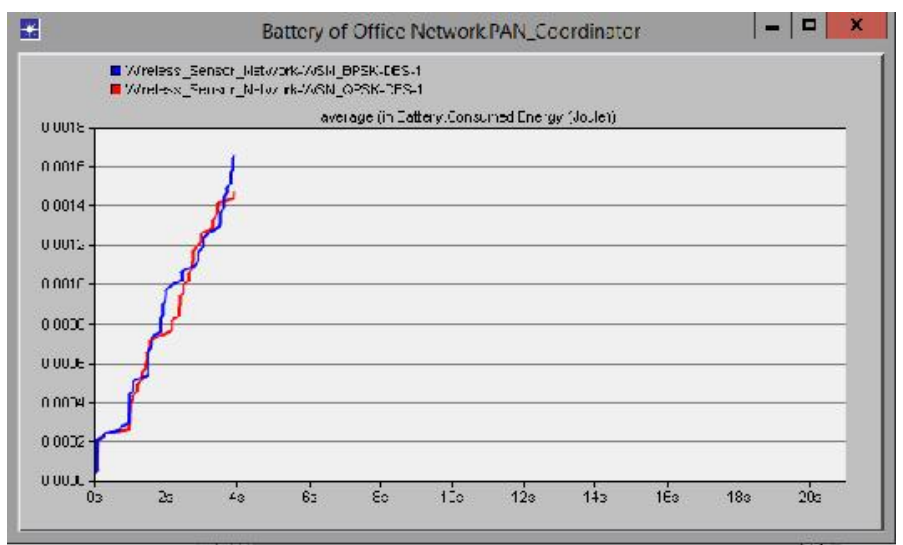

Figure 6. Energy Consumed by the PAN_Coordinator 


\subsubsection{GTS End Device}

Figure 7 shows energy consumed by the GTS End Device. It has been observed that it is 0.00129715 and 0.00150752 joules for QPSK and BPSK respectively. It has been experimentally proved that energy consumed is minimum in case of QPSK while it is maximum in case of BPSK.

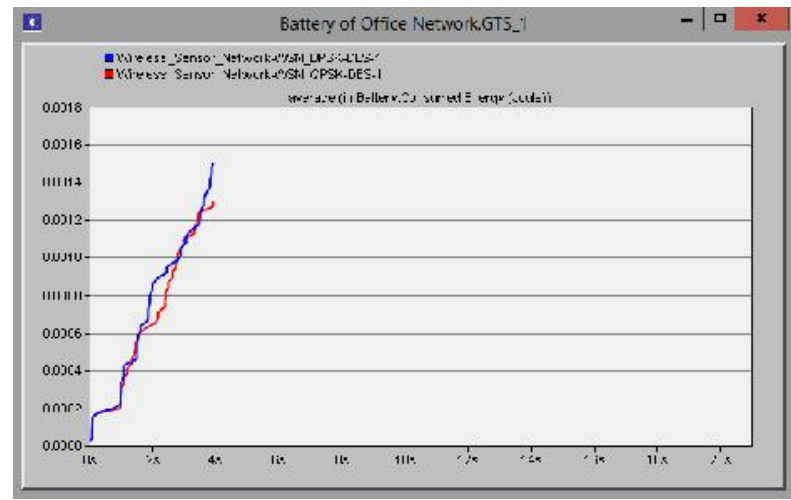

Figure 7. Energy Consumed by the GTS Device

\subsubsection{CAP End Device}

Figure 8 reveals that battery energy consumed by the Non GTS End Device is 0.00145471 and 0.00177974 joules respectively for QPSK and BPSK. It is observed that energy consumed is minimum in case of QPSK in comparaison with BPSK modulation.

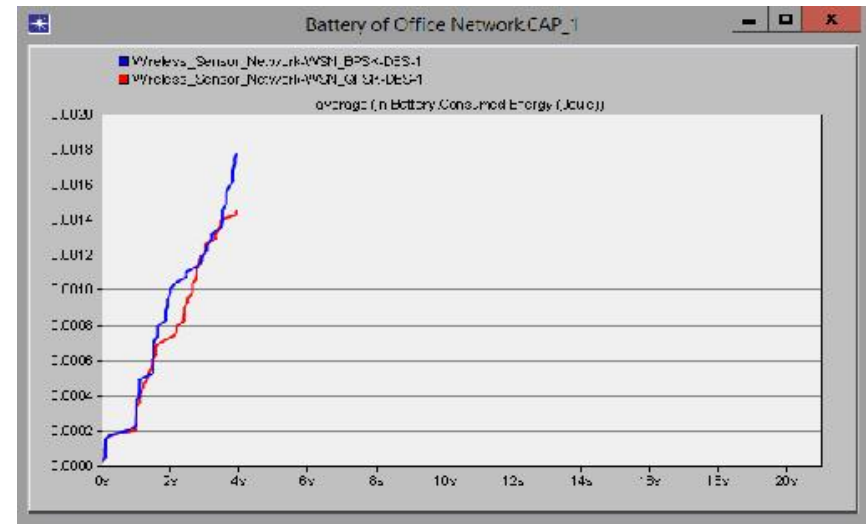

Figure 8. Energy Consumed by the Non GTS End Device

\subsection{Radio Receiver Power}

\subsubsection{PAN Coordinator}

Figure 9 below shows the power received by the radio receiver at the PAN Coordinator is 0.00023363 and 0.00023844 watts for BPSK and QPSK respectively. Her it is observed that power received is almost the same in case of BPSK and QPSK because the two modulation schemes receives power according to A2/2 where $A$ is amplitude. 


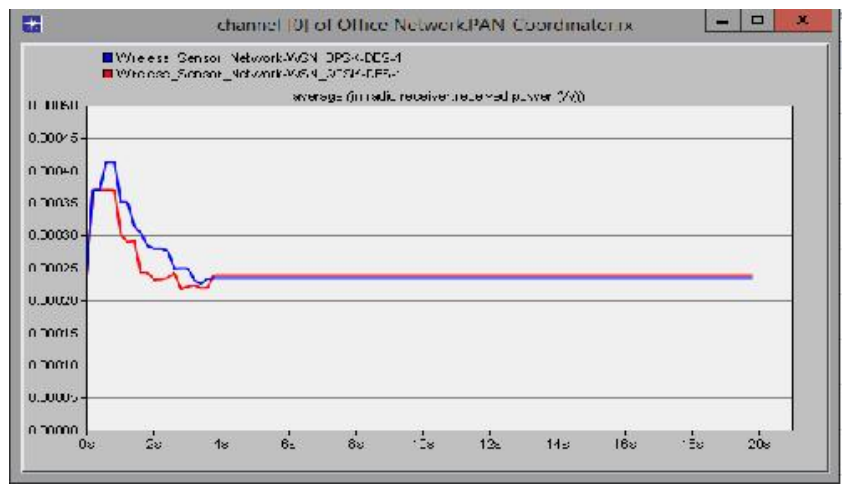

Figure 9. Received Power at the PAN Coordinator

\subsubsection{GTS End Device}

Figure 10 indicates that the power received at GTS End Device is $2.9762 .10^{-6}$ and 3.4014.10-6 watts for BPSK and QPSK respectively.

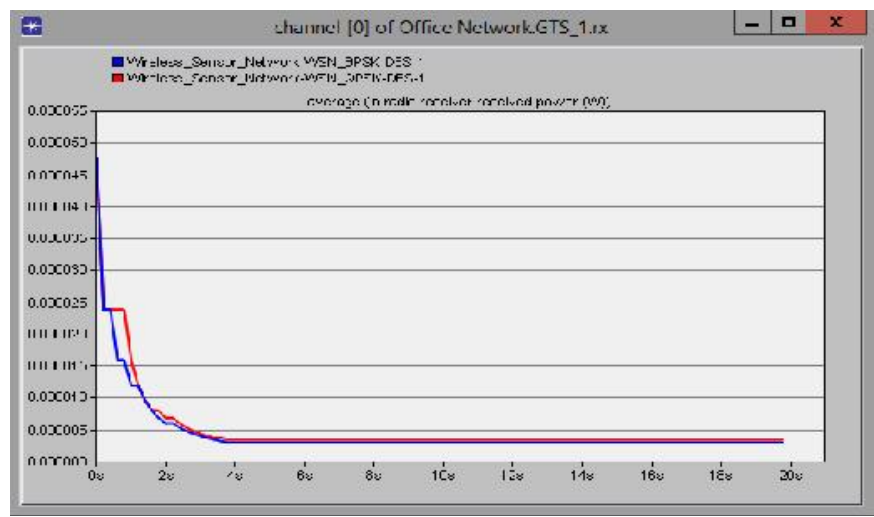

Figure 10. Received Power at the GTS End Device

\subsubsection{CAP End Device}

Figure 11 indicates the power received at the Non GTS End Device. It has been observed that it is $9.2828 .10^{-5}$ and $7.594 .10^{-5}$ watts for BPSK and QPSK respectively. It has beeninvestigated that power received is maximum in case of BPSKwhile it is minimum in case of QPSK.

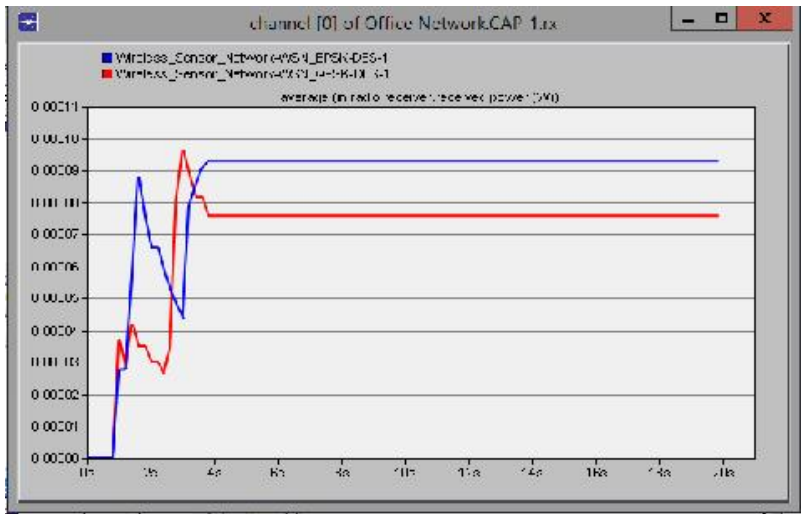

Figure 11. Received Power at the Non GTS End Device

TELKOMNIKA Vol. 16, No. 1, October 2015 : 98 - 105 


\section{Conclusion}

This paper presentsthe network output load, energy consumption and power reception for a WPAN wireless sensor network using OPNETModeler 14.5. Here two different modulation scenarios for BPSK and QPSK have been considered.

Results reveals that the network output load given by the BPSK modulation is higher than that of the QPSK modulation in the case of PAN_Coordinator and GTS end device and lower than the QPSK modulation in the case of CAP end device(Non GTS end device).

Also the energy consumed by BPSK modulation is higher than QPSK modulation for all types of devices in 802.15.4 wireless sensor networks .

Further the power received by the radio receiver given by the BPSK modulation is higher than that of the QPSK modulation in the case of the CAP end device (Non GTS end device) and lower than the QPSK modulation in the case of PAN_Coordinator and GTS end device.

\section{References}

[1] Jurcik P, Koubaa A, Alves M, Tovar E, Hanzalek Z. A Simulation Model for the IEEE 802.15.4 protocol: Delay/Throughput Evaluation of the GTS Mechanism. Modeling, Analysis, and Simulation of Computer and Telecommunication Systems, 2007. MASCOTS '07. 15th International Symposium. 2007.

[2] Paul Daniel Mitchell, Jian Qiu, Hengguang Li, David Grace. Use of aerial platforms for energy efficient medium access control in wireless sensor networks. Computer Communications. 2010.

[3] SM Mahdi Alavi, Michael J Walsh, Martin J Hayes. Robust distributed active power control technique for IEEE 802.15.4 wireless sensor networks - A quantative feedback theory approach. Control Engineering Practice. 2009.

[4] David Coudert, Napoleao Nepomuceno, Herve Rivano. Power-efficient radio configuration in fixed broadband wireless networks. Computer Communications. 2010.

[5] Patrick Hosein. Adaptive Algorithm for Mapping Channel Quality Information to Modulation and Coding Schemes. IEEE. 2009.

[6] Mohamed Nazim Abdeddaim. Analyse des performances d'un réseau de capteurs exploitant le standard IEEE 802.15.4. Thèse doctorat. 2012.

[7] Mayyada Hammoshi, AwnySayed. An analysis for a cluster tree zigbee topology. Journal of Theoretical and Applied Information Technology. 2014; 64(3).

[8] Prasad P Netalkar, Yasha Kaushal, Dr N Shekar V Shet. Zigbee Based Wireless Sensor Networks for Smart Campus. International Journal Of Modern Engineering Research (IJMER). 2014; 4(7).

[9] Devender, Chirag, Kuldeep Vats, Deepika Madaan. WSN Based Performance Evaluation of Coordinator Fails in Different PAN Network using OPNET. International Journal of Computer Science and Mobile Computing. 2014; 3(11): 504-513.

[10] Devender, Chirag, Kuldeep Vats, Deepika Madaan. WSN Based Performance Evaluation of Coordinator Fails in Different PAN Network using OPNET. International Journal of Computer Science and Mobile Computing. 2014; 3(11): 504-513.

[11] MM Chandane, SG Bhirud, SV Bonde. Performance Analysis of IEEE 802.15.4. International Journal of Computer Applications. 2012; 40(5).

[12] Singh K. Optimisation of IEEE 802.15.4 for suburban smart grid communication in hybrid LTE-WSN network. Master's thesis. University of Oulu, Department of Communications Engineering, Master's Degree program in Wireless Communications Engineering. 2014: 57. 